\title{
The role of genetic factors in carpal tunnel syndrome etiology: A review
}

\author{
Andrzej Żyluk ${ }^{A-F}$ \\ Clinic of General and Hand Surgery, Pomeranian Medical University in Szczecin, Poland \\ A - research concept and design; B - collection and/or assembly of data; $C$ - data analysis and interpretation; \\ $\mathrm{D}$ - writing the article; $\mathrm{E}$ - critical revision of the article; $\mathrm{F}$ - final approval of the article
}

Address for correspondence

Andrzej Żyluk

E-mail:azyluk@hotmail.com

\section{Funding sources \\ None declared}

Conflict of interest

None declared

Received on May 6, 2019

Reviewed on December 12, 2019

Accepted on March 10, 2020

Published online on May 14, 2020

\begin{abstract}
The direct causes of idiopathic carpal tunnel syndrome (CTS) still remain obscure. It has been suggested that the pathology of tendons and other connective tissue structures within the carpal tunnel may be involved in its etiology. The objective of this study was to review the literature about the potential role of genetic factors in the etiology of CTS. Three different mechanisms are suspected to be involved in genetic predisposition to CTS: collagen synthesis, collagen degradation and protection against oxidative stress effect in connective tissue. Several gene groups are involved in the regulation and modulation of these mechanisms, and the research reviewed in this study showed their possible effect on the development of CTS. Variants within the COLTA7, COLSA7 and COL71A7 genes - encoding the synthesis of minor collagen subtypes - may potentially be involved, as they alter the mechanical properties of tendons and other connective tissue structures within the carpal tunnel. The collagen within connective tissue structures is also remodeled by matrix metalloproteinases (MMPs), so variants of these genes have also been investigated for their possible role in the risk of CTS development. Next, the variants of genes encoding glutathione S-transferase (GST) synthesis were found to be involved in the etiology of CTS. The findings from the abovementioned studies provide reliable information on the potential role of genetic risk factors in the development of CTS.
\end{abstract}

Key words: genetic factors, collagen synthesis, gene variants, carpal tunnel syndrome etiology

Cite as

Żyluk A. The role of genetic factors in carpal tunnel syndrome etiology: A review. Adv Clin Exp Med. 2020;29(5):623-628. doi:10.17219/acem/118846

DOI

10.17219/acem/118846

\section{Copyright}

Copyright by Author(s)

This is an article distributed under the terms of the

Creative Commons Attribution 3.0 Unported (CC BY 3.0)

(https://creativecommons.org/licenses/by/3.0/) 


\section{Introduction}

Carpal tunnel syndrome (CTS) is the most common compression neuropathy in the upper limbs. Mechanical compression and local ischemia result in symptoms of paresthesia (numbness and tingling), pain, and sensory and motoric disturbance along the median nerve. ${ }^{1}$ The pathogenesis of most cases of CTS has not been determined. In the "idiopathic" syndrome, the direct cause of the increased pressure in the carpal tunnel is unknown. Among numerous concepts on pathogenesis of CTS, the possible involvement of tendons and/or other connective tissue structures within the carpal tunnel structure has been proposed..$^{2-4}$ Considering the proximity of the 9 flexor tendons and the thick flexor retinaculum to the median nerve within the limited space of the carpal tunnel, it is not unlikely that pathology of these tendons or the retinaculum may contribute to CTS pathology. This concerns possible genetic factors which may influence the characteristics and regulation of collagen fibrils, which are the basic ingredient of connective tissue structures such as tendons, ligaments and bones.

Collagen is not a homogenous substance. Several subtypes of collagen have been identified, but type I is the one most commonly found in all connective tissue structures. Although the collagen fibril consists predominantly of type I collagen, several other quantitatively minor collagens, including types V, XI and XII, have been identified. These types were suspected as playing a possible role in regulating the formation and maintaining the structural integrity of the collagen fibril and surrounding matrix. ${ }^{2}$ It has also been postulated that collagen types $\mathrm{V}$ and $\mathrm{XI}$ interact to regulate fibrillogenesis (the size and assembly of fibrils) during tendon development. ${ }^{5}$ Characteristic features of collagen, such as elasticity and endurance, may be related to the proportions of the minor types of collagen composing the fibrils of tendons and filaments. Changes in these properties may translate into modulation of pressure in the carpal tunnel, followed by the development of compression of the median nerve. Previous studies showed a relationship between variants in genes encoding the production and degradation of collagen and some inflammatory/overuse diseases of the connective tissue and increased risk of traumatic damage to the tendons and ligaments. ${ }^{6-10}$ The results of these studies prompted investigators to search for a possible association between selected gene variants and the risk of developing CTS.

Another mechanism which may be involved in the etiology of CTS is the activity of matrix metalloproteinase (MMP) genes. ${ }^{11}$ It has been shown that the products of these genes play an important role in connective tissue remodeling through collagen fibril degradation. Previous studies showed an association between variants of these genes and tendon and ligament diseases, as well as susceptibility to tendon and ligament injury. ${ }^{12-16}$
Oxidative stress is another mechanism suspected to be involved in the etiology of CTS. It was found to play a role in the development of systemic inflammatory diseases, diabetes and some malignancies. Previous research showed that oxidative stress as a result of overproduction of reactive oxygen and hydroxyl free radicals in synovial connective tissue around the carpal tunnel may result in tissue damage, edema and subsequent development of compression of the median nerve. ${ }^{17}$ Glutathione S-transferases (GST) are a large family of isoenzymes involved in the defense mechanisms against reactive oxidative stress. Variants in genes encoding production of these proteins were investigated as playing a possible role in the development of CTS. ${ }^{18}$

The objective of this study was to review the literature on the potential role of genetic factors in the etiology of CTS.

\section{Material and methods}

This article presents a review of the published literature from PubMed and MEDLINE databases on the role of genetic factors in the etiology of CTS. A search for Cochrane Reviews was performed, but we were unable to identify any relevant reviews on this topic. The keywords used when searching for articles were as follows: carpal tunnel pathomechanism, genetics, collagen synthesis, polymorphism, molecular mechanisms, COL genes, MMP genes, and oxidative stress. We attempted to ascertain the role of selected factors - such as collagen, MMP and GST gene variants - as risk factors in the development of CTS.

\section{Results}

\section{The role of collagen gene variants}

Previous studies have found that variants within the genes encoding for synthesis of collagen types I, V, XI, and XII may be implicated in their potential role in various musculoskeletal soft tissue disorders. It was shown that a variant within $C O L 1 A 1$, which encodes for $\alpha$ 1chain of type I collagen of type I collagen, is the functional Sp1 binding site polymorphism (rs1800012, G/T). The TT genotype was associated with the mechanical properties of tendons and ligaments, specifically with greater endurance of the anterior cruciate ligament in the knee joint. ${ }^{8}$ Likewise, variants of the COL5A1 (rs71746744) and COL11A1 (rs3753841, T/C and rs1676486, C/T) genes - encoding for type $\mathrm{V}$ and XI collagen - are involved in modulating the risk of chronic Achilles tendinopathy. ${ }^{7}$ The results of these studies prompted investigators to search for potential associations between variants of genes encoding for synthesis of minor collagen types and the risk of CTS development. 
The following associations were found:

1. The presence of the $\mathrm{T}$ allele of the COL11A1 gene (rs1676486) has been reported to be associated with decreased $\alpha 1(\mathrm{XI})$ collagen chain production, and by implication type XI collagen synthesis might be involved in the etiology of CTS.7,10

2. The presence of T-C (AGGG) variants (rs3753841, rs1676486 and rs1746744) of the COL5A1 and COL11A1 genes is associated with altered mRNA stability, which results in altered type $\mathrm{V}$ and XI collagen synthesis. Both types of collagen regulate collagen fibril assembly and diameter; thus, these variants could alter the mechanical properties of tendons and other connective tissue structures within the carpal tunnel, which may be implicated in the etiology of CTS. ${ }^{3,7}$

3. A significant association has been found between the single-nucleotide polymorphism TT of COL11A1 (rs3753841, T/C) and increased risk of CTS development. This variant, located in exon 52, results in a non-synonymous amino acid exchange of leucine to proline at position 1323 of the $\alpha 1(\mathrm{XI})$ chain, whereas the rs1676486 variant located in exon 62 results in amino acid substitution from proline to serine at position 1535 . The combination of alleles from these variants could potentially cause conformational changes in type XI collagen with a potential effect on the structural and functional properties of new collagen fibrils. These changes, through their effect on tendons and other connective tissue structures in the carpal tunnel, may be further implicated in the etiology of CTS. ${ }^{10}$

4. The abovementioned minor $\mathrm{T}$ allele variant of COL1A1 (rs1800012, G/T), which encodes for the $\alpha 1$ chain of type I collagen, was significantly associated with an increased risk of CTS among women. The substitution of a tyrosine with a guanine nucleotide within the Sp1 binding site of intron 1 of COL1A1 has been suggested to result in an increased binding affinity for transcription factor Sp1. This results in COL1A1 gene expression and the overproduction of type I collagen homotrimers consisting of $3 \alpha 1$ chains. Increased amounts of type I collagen homotrimers in tendons and other connective tissue structures is believed to change their mechanical characteristics in terms of susceptibility to injury. These changes may also be implicated in the development of increased pressure in the carpal tunnel. , $^{3,6}$

Dada et al. reported the results of their genetic studies performed on a self-reported Colored South African population. One hundred and three participants with a history of carpal tunnel release surgery and 150 matched control participants, without any reported history of CTS symptoms, were genotyped for COL1A1 rs1800012 (G/T), COL11A1 rs3753841 (T/C), COL11A1 rs1676486(C/T), COL11A2 rs1799907 (T/A), and COL12A1 rs970547 (A/G). It was shown that the TT variant of COL11A1 rs 3753841 was statistically significantly overrepresented among CTS patients in comparison with the control group $(21.4 \%$ vs $7.9 \% ; \mathrm{p}=0.004)$. A trend for the $\mathrm{T}$ minor allele to be overrepresented in the CTS group ( $\mathrm{p}=0.055)$ was also observed with statistically significant differences ( $p=0.036$ ) when only women were considered in the analysis. The authors believe that constructed inferred variants, including the previously mentioned COL5A1 gene variant rs71746744 (AGGG), suggest that gene-gene interactions between COL5A1 and COL11A1 modulate the risk of developing CTS. ${ }^{3}$

Findings from the abovementioned studies provide reliable information on the potential role of genetic risk factors and the possible role of variations of collagen fibril composition in the etiology of carpal tunnel syndrome. Genetic factors can potentially be included in models developed to identify individuals at risk of CTS.

\section{The effect of variants of MMP genes on chromosome 11q22}

In the previous paragraph, the association between some variants of genes encoding the synthesis of collagen and the risk of developing CTS was discussed. However, the final properties of collagen are also related to other factors. The collagen fibrils within connective tissue structures are remodeled by a family of endopeptidases called MMPs, ${ }^{11}$ among other proteins. The following roles of MMPs in the modulation of collagen degradation have been identified ${ }^{12-15}$ :

- MMP3 and MMP10 are involved in the degradation of several collagens, including type $\mathrm{V}$, proteoglycans and other extracellular matrix proteins.

- These MMPs also have the potential to activate several pro-MMPs.

- MMP1 is responsible for degrading the triple-helical region in most collagens.

- MMP12 is responsible for degrading elastin and other extracellular matrix proteins.

Several studies have reported a significant association of $M M P 3$ gene variants with chronic Achilles tendinopathy: AA MMP3 rs679620 (A/G, E45 K), CC MMP3 rs591058 (T/C) and $A A M M P 3$ rs650108 (G/A). ${ }^{13}$ An inferred haplotype constructed from MMP variants $M M P 10$ rs486055 (C/T, R5 3), MMP1 rs1799750 (G/GG), MMP3 rs679620 (A/G), and MMP12 rs2276109 (A/G), which are clustered together on chromosome 11q22, was found to be associated with modulation of the risk of anterior cruciate ligament ruptures in the knee joint. ${ }^{14,16}$ Considering the potential effect of MMPs on homeostasis of soft tissue and their structures (tendons and ligaments), studies were commenced to investigate a possible association between various MMP variants and the risk of CTS development. The 4 variants of MMPs were particularly suspected because of previously reported associations with other musculoskeletal diseases and because of their potential functions - the ability to degrade various types of collagens. The MMP1 rs1799750 (G/GG) variant consists of the presence (GG) or absence (G) of an extra guanine 
nucleotide at position $1607 \mathrm{bp}$. The GG variant creates an Ets binding site that leads to increased transcription of the MMP1 gene compared to the allele without an extra G nucleotide. The MMP12 rs2276109 (A/G) variant modulates the binding of the transcription factor activator AP-1 protein, which regulates MMP12 expression. Variants MMP3 rs679620 and MMP10 rs486055 result in a single-nucleotide substitution that causes an amino acid change in proteins - the products of these genes. ${ }^{11,15}$ The specific consequences of these amino acid substitutions are unknown, but previous studies have suggested potential associations with tendon and ligament diseases, susceptibility to tendon and ligament injuries, and the risk of CTS development.

Burger et al. reported the results of genetic studies on a self-reported Colored South African population: 97 women with clinically and electrophysiologically confirmed CTS and 131 healthy volunteers without any history of CTS symptoms were genotyped for 4 MMP gene variants: $M M P 10$ rs486055 (C/T), MMP1 rs1799750 (G/GG), MMP3 rs679620 (A/G), and MMP12 rs2276109 (A/G). The results of this genotyping showed no independent association between these gene variants and CTS (negative outcome). Furthermore, no significant association was found between any of the inferred haplotypes constructed from these variants and CTS. ${ }^{12}$ However, this finding does not exclude other variants within the same or other MMP genes within this locus from potentially being associated with the risk of CTS. Several other MMP genes, including $M M P 7, M M P 8, M M P 13$, and $M M P 20$, are located on the long arm of chromosome 11 , close to the previously investigated genes. The MMP3 5A/6A variant has been associated with several multifactorial conditions and was found to be involved in the regulation of MMP gene expression. ${ }^{16}$ The authors suggest that these gene variants may be reasonable CTS candidates for future studies. Also, the use of next-generation sequencing technologies, i.e., targeted sequencing, in identifying additional potentially associated variants within the MMP cluster may yield positive results. It should be noted that the abovementioned study was based on a specific population and that the number of participants was relatively small. Therefore, further studies of other variants of the roles of MMP genes in modulating the risk of development of CTS, based on a European population and involving a greater cohort of patients, seems to be justified.

\section{The role of GST variants}

Another mechanism suspected to be involved in the development of idiopathic CTS is oxidative stress as a result of overproduction of free oxygen (.O) and hydroxyl $(\cdot \mathrm{OH})$ radicals in the sub-synovial soft tissue of tendons and ligaments. ${ }^{17,18}$ Increased synthesis or insufficient elimination of reactive oxygen species (ROS) may cause damage to soft tissue structures and may be an underlying mechanism in the development of systemic inflammatory diseases and malignancies. There are several substances known as antioxidants or radical scavengers which exhibit properties that eliminate free radicals, including vitamins (C, D and E), amino acids (N-acetylcysteine), hormones (steroids), and mannitol. There are also several enzymes with this potential, such as peroxidase, catalase and GSTs (oddly enough, hydroxyl radicals do not have their own natural detoxifying mechanisms). The GSTs are a large family of isoenzymes involved in the defense mechanism against the cytotoxic activity of ROS by inactivating their secondary metabolites. The most investigated isoenzyme members of this family are GST-mu1 (GSTM1), GST-theta1 (GSTT1) and GST-pi1 (GSTP1). They are suspected of playing a protective role against oxidative stress-related development of systemic inflammatory diseases and malignancies. ${ }^{19}$ The most common GSTM1 and GSTT1 polymorphisms are deletions in these genes, resulting in a lack of enzyme function. Another frequent polymorphism in this group is GSTP1 Ile105Val, of which the Ile105Val/Val (AG) and Ile105Val/Val (GG) variants result in reduced enzymatic activity in comparison with that of the Ile105Val/Val (AA) variant. ${ }^{20}$ Several studies have demonstrated an association between some GST family isoenzyme variants and rheumatoid diseases, diabetes and malignancies in whose pathogenesis ROS are involved. ${ }^{19}$ The results of these studies prompted investigators to search for potential associations between variants of GST genes and the risk of developing CTS.

Eroğlu et al. reported the results of their study of the incidence of 3 GST variants - GSTM1, GSTT1 and GSTP1 Ile105Val - in a Turkish population of CTS patients and controls ( $\mathrm{n}=140$ and $\mathrm{n}=97$, respectively). The incidence of GSTT1 and GSTM1 variants was determined using the polymerase chain reaction (PCR) method, whereas PCRrestriction fragment length polymorphism (PCR-RFLP) was used to detect the GSTP1 Ile105Val variant. The researchers found a statistically significant $(\mathrm{p}=0.01)$ higher incidence of the GSTM1-null variant in CTS patients compared to the healthy controls. Moreover, the GSTM1-null variant was associated with an approximately twofold increase in the risk of CTS. The combination of the GSTM1-null and GSTT1 variants was also more prevalent in CTS patients, but it was of borderline statistical significance $(\mathrm{p}=0.045)$ and did not increase the risk of CTS development. The authors also reported a statistically significant association between greater clinical severity of CTS (in terms of higher Levine questionnaire scores) in patients with the GSTP1 Ile/Val and Val/Val variants compared to GSTP1 Ile/Ile, which was more frequent in patients with clinically milder disease. ${ }^{18}$ The authors suggest that future studies investigating the potential role of GST variants in the development of CTS are justified.

Fernandez-de-Las-Penas et al. investigated the association of the Val158Met polymorphism with treatment outcomes in 120 female patients with CTS who received either surgery or manual therapy. The patients were randomly allocated to the treatment methods: 60 patients received 
3 sessions of manual therapy and 60 underwent carpal tunnel release $(n=60)$. The $r 44680$ genotypes were determined after amplifying the Val158Met polymorphism with PCR. The subjects were classified according to their Val158Met polymorphism: Val/Val, Val/Met or Met/Met. Final outcomes were assessed 12 months after the intervention. No interaction was observed between the Val158Met genotype and any outcome: pain, symptom or function severity. ${ }^{21}$

Cevik et al. investigated the influence of interleukin 1 (IL-1) receptor antagonist (IL-1Ra) and angiotensin-converting enzyme (ACE) I/D polymorphisms on the susceptibility to CTS in 158 patients and 151 healthy controls. No statistically significant association was found between gene polymorphisms and the risk of developing CTS. ${ }^{22}$

\section{Discussion}

Three different mechanisms are thought to be involved in genetic predisposition to CTS: collagen synthesis, collagen degradation and protection against the effects of oxidative stress in connective tissue. Several gene groups are involved in the regulation and modulation of these mechanisms and the studies presented herein demonstrated that they may be associated with the etiology of CTS. There are some interesting aspects of the disease which suggest the potential effect of genetic predispositions to its development ${ }^{23-25}$ :

- early onset of CTS in adolescents and young adults;

- familial occurrence - most of the members of the family have been affected;

- familial occurrence - higher incidence of the condition among relatives of some patients than in the general population;

- bilateral manifestation of CTS.

None of studies conducted to date have dealt with these specific aspects of the syndrome; therefore, additional investigations in this field seem to be promising. Another unexplored aspect of the genetic predisposition to CTS is that most studies have been based on non-European populations (South African, Turkish and Brazilian). It is known that the incidence of specific mutations may differ among races, so investigation into the abovementioned associations between selected gene variants and the risk of CTS in Caucasians seems to be justified.

\section{Conclusions}

The findings from the studies reviewed in this paper provide reliable information on the potential role of genetic risk factors in the development of CTS. None of the studies conducted to date have dealt with these specific aspects of the syndrome, thus additional studies in this field seem to be appropriate.

\section{ORCID iDs}

Andrzej Żyluk (1) https://orcid.org/0000-0002-8299-4525

\section{References}

1. Puchalski P, Zyluk P, Szlosser Z, Zyluk A. Factors involving the clinical profile of carpal tunnel syndrome. Handchir Mikrochir Plast Chir. 2018;50(1):8-13.

2. Burger $\mathrm{M}$, de Wet $\mathrm{H}$, Collins $\mathrm{M}$. The $B G N$ and $A C A N$ genes and carpal tunnel syndrome. Gene. 2014;551(2):160-166.

3. Dada S, Burger MC, Massij F, de Wet H, Collins M. Carpal tunnel syndrome: The role of collagen gene variants. Gene. 2016;587(1):53-58. doi:10.1016/j.gene.2016.04.030

4. Burger $\mathrm{M}$, de Wet $\mathrm{H}$, Collins $\mathrm{M}$. The COL5A1 gene is associated with increased risk of carpal tunnel syndrome. Clin Rheumatol. 2014;34(4): 767-774.

5. Wenstrup RJ, Smith SM, Florer JB, et al. Regulation of collagen fibril nucleation and initial fibril assembly involves coordinate interactions with collagens $\mathrm{V}$ and $\mathrm{XI}$ in developing tendon. J Biol Chem. 2011; 286(23):20455-20465.

6. Ficek K, Cieszyk O, Kaczmarczyk M, et al. Gene variants within the COL1A1 gene are associated with reduced $A C L$ injury in professional soccer players. J Sci Med Sport. 2013;16(5):396-400.

7. Hay M, Patricos J, Collins R, et al. Association of type XI collagen genes with chronic Achilles tendinopathy in independent populations form South Africa and Australia. Br J Sports Med. 2013;47(9):569-574.

8. Posthumus M, September AV, Keegan M, et al. Genetic risk factors for anterior cruciate ligament ruptures: COL1A1 gene variant. Br J Sports Med. 2009;43(5):352-356.

9. Posthumus $M$, September AV, O'Cuinneagain D, van der Merwe W, Schwellnus MP, Collins M. The association between the COL12A1 gene and anterior cruciate ligament ruptures. Br JSports Med. 2010;44(16): 1160-1165.

10. Mio F, Chiba K, Hirose $Y$, et al. A functional polymorphism in COL11A1, which encodes the alpha 1 chain of type $\mathrm{XI}$ collagen, is associated with susceptibility to lumbar disc herniation. Am J Hum Genet. 2007: 81(6):1271-1277.

11. Pasternak B, Aspenberg P. Metalloproteinases and their inhibitors: Diagnostic and therapeutic opportunities in orthopedics. Acta Orthop. 2009;80(6):693-703.

12. Burger MC, De Wet $\mathrm{H}$, Collins M. Matrix metalloproteinase genes on chromosome 11q22 and risk of carpal tunnel syndrome. Rheumatol Int. 2016;36(3):413-419. doi:10.1007/s00296-015-3385-z

13. Raleigh SM, van der Merwe L, Ribbans WJ, Smith RK, Schwellnus MP, Collins M. Variants within the MMP3 gene are associated with Achilles tendinopathy: Possible interaction with the COL5A1 gene. Br J Sport Med. 2009;43(7):514-520.

14. Posthumus $M$, Collins $M$, van der Merwe $L$, et al. Matrix metalloproteinase genes on chromosome $11 \mathrm{q} 22$ and the risk of $\mathrm{ACL}$ rupture. Scand J Med Sci Sport. 2012;22(4):523-533.

15. Somerville RP, Oblader SA, Aptek SS. Matrix metalloproteinases: Old dogs with new tricks. Genome Biol. 2003;4(6):216. doi:10.1186/ gb-2003-4-6-216

16. Malila S, Yuktanandana P, Saowaprut S, Jiamjarasrangsi W, Honsawek S. Association between matrix metalloproteinase-3 polymorphism and ACL ruptures. Genet Mol Res. 2011;10(4):4158-4165.

17. Kim JK, Koh YD, Hann HJ, Kim MJ. Oxidative stress in sub-synovial connective tissue of idiopathic carpal tunnel syndrome. J Orthop Res. 2010;28(11):1463-1468.

18. Eroğlu P, Erkol İnal E, Sağ ŞÖ, Görükmez Ö, Topak A, Yakut T. Associations analysis of GSTM1, T1 and P1 lle105Val polymorphisms with carpal tunnel syndrome. Clin Rheumatol. 2016;35(5):1245-1251. doi:10. 1007/s10067-014-2855-0

19. Board PG, Menon D. Glutathione transferases, regulator of cellular metabolism and physiology. Biochem Biophys Acta. 2013;1830(5): 3267-3288.

20. Ali-Osman F, Akande O, Antoun G, Mao XJ, Buolamwini J. Molecular cloning, characterization and expression in Escherichia coli of full-length cDNAs of three human GSTP1 variants: Evidence of catalytic activity of encoded proteins. J Biol Chem. 1007;272(15): 10004-10012. 
21. Fernandez-de-Las-Penas C, Ambite-Quesada S, Fahandezh-Saddi Díaz H, Paras-Bravo P, Palacios-Cena D, Cuadrado ML. The Val158Met polymorphism of the catechol-O-methyltransference gene is not associated with long-term treatment outcomes in carpal tunnel syndrome: A randomized clinical trial. PLoS One. 2018;13(10):e0205516. doi:10.1371/journal.pone.0205516

22. Cevik B, Tekcan A, Inanir A, Kurt SG, Yigit S. The investigation of association between IL-1Ra and ACE I/D polymorphisms in carpal tunnel syndrome. J Clin Lab Anal. 2018;32(1):e22204. doi:10.1002/jcla.22204
23. Puchalski P, Szlosser Z, Zyluk A. Familial occurrence of carpal tunnel syndrome. Neurol Neurochir Pol. 2019;53(1):43-46. doi:105603/PJNNS. a2019.0004

24. Gossett JG, Chance PF. Is there a familial carpal tunnel syndrome? An evaluation and literature review. Muscle Nerve. 1998;21(11):1533-1536.

25. Alford JW, Weiss AP, Akelman E. The familial incidence of carpal tunnel syndrome in patients with unilateral and bilateral disease. AmJ Orthop (Belle Mead NJ). 2004;33(8):397-400. 